\title{
Safety and advantage of no drain policy after thoracoscopic major lung resection
}

\author{
Kazuhiro Ueda \\ Department of General Thoracic Surgery, Graduate School of Medical and Dental Sciences, Kagoshima University, Kagoshima, Japan \\ Correspondence to: Kazuhiro Ueda. Department of General Thoracic Surgery, Graduate School of Medical and Dental Sciences, Kagoshima \\ University, 8-35-1 Sakuragaoka, Kagoshima 890-8520, Japan. Email: k7433286@kadai.jp. \\ Provenance and Peer Review: This is an invited article commissioned and reviewed by the Section Editor Shuangjiang Li (Department of Thoracic \\ Surgery and West China Medical Center, West China Hospital, Sichuan University, Chengdu, China). \\ Response to: Miyazaki T, Nagayasu T. No drain policy for "ultimate” enhanced recovery after surgery. J Thorac Dis 2019;11:S1900-2. \\ Li P, Li S, Che G. Role of chest tube drainage in physical function after thoracoscopic lung resection. J Thorac Dis 2019;11:S1947-50.
}

Submitted Nov 21, 2019. Accepted for publication Dec 22, 2019.

doi: $10.21037 /$ jtd.2019.12.61

View this article at: http://dx.doi.org/10.21037/jtd.2019.12.61

It is my great honor to express my opinion on the editorial commentary $(1,2)$ regarding our paper, "No drain after thoracoscopic major lung resection for cancer helps preserve the physical function" (3). First of all, we could achieve a satisfactory outcome with our no drain strategy after major lung resection for cancer (4), partly because of comprehensive coverage by the national medical insurance system in Japan: most of the materials to seal air leaks, such as endostaplers, fibrin sealant, and bioabsorbable mesh, are covered by the Japanese national insurance system. However, the technical details on the use of these materials are still not standardized and they are used differently by each thoracic surgeon in Japan. Thus, we believe that learning some technical tips and using ideal devices to deliver fibrin sealant are necessary to achieve firm attachment between the bioabsorbable mesh and the injured lung parenchyma. We believe that the length of postoperative chest tube placement should be shortened in a step-by-step manner in accordance with the surgeon's technical maturation.

An additional determinant of a successful no drain strategy is the accurate identification of patients, at the removal of the tracheal tube, who have no substantial need for chest tubes after surgery. Careful observation during the intraoperative confirmation test and a water seal test are mandatory (4).

Nonetheless, one may have concerns as to whether the no drain strategy was really verified. In our previous study that included 102 patients who did not undergo postoperative chest tube placement, no patients required chest tube reinsertion for air leak or subcutaneous emphysema (4). However, we experienced two patients who required chest tube reinsertion among our subsequent patients who were managed by the no drain strategy. One patient developed delayed air leak on postoperative day 7 . However, the chest tube reinsertion in this case was not induced by the no drain strategy because chest tube reinsertion was unavoidable even though the patient had been managed by the standard drainage strategy. Another patient developed ipsilateral lung collapse on the day after robotic-assisted lobectomy, which spontaneously resolved after chest tube reinsertion. We believe that changing the surgical approach can alter the technical quality of the intraoperative sealing of air leaks, as well as increase the incidence of iatrogenic organ injury, particularly during robotic-assisted lung surgery (5). We therefore believe that a no drain strategy should be implemented in operations with surgeon using his most comfortable approach. Another concern may be the potential for postoperative intrathoracic bleeding. We believe that the most frequent site of bleeding may be derived from chest wall incision. Thus, we apply a no drain policy in cases involving thoracoscopic major lung resection, but not open thoracotomy. We also recommend the placement of chest tubes in patients undergoing vasculoplasty of the major pulmonary vessels or repairing for major vessel injury. Finally, chylothorax can also be 
a reason for chest tube reinsertion. Fortunately, we have never experienced chest tube reinsertion for postoperative chylothorax, despite basically performing mediastinal lymph node dissection for patients with primary lung cancer. Because the branch of the thoracic duct is located at the designated site, careful clipping or dividing with sealing devices in this area could contribute to reducing the incidence of postoperative chylothorax.

The main results of our paper were that the no drain strategy contributed to the reduction of pain, the preservation of the ventilatory capacity, and the preservation of the exercise capacity in the early postoperative period in patients undergoing thoracoscopic major lung resection for cancer (3). Unfortunately, the advantage of no drain management on pain reduction and functional preservation disappeared on postoperative day 7. However, we expect that a no drain strategy can contribute to reducing the incidence of postoperative cardiopulmonary complications by promoting early mobilization of patients after surgery. Nevertheless, one may still be concerned about the adverse effects of no drain management on the late-postoperative pulmonary function. It has been claimed that the absence of suctioning via drain results in fluid collection and prevents lung expansion, which eventually prevents the latepostoperative recovery of the pulmonary function. We do not agree with this suggestion because we rarely see fluid collection in the late-postoperative period in patients managed by our no drain strategy. However, it is important to compare the extent of expansion in the ipsilateral lung in the late-postoperative period among different institutes that follow their own management strategies regarding chest tube drainage after major lung resection.

\section{Acknowledgments}

Funding: None.

Cite this article as: Ueda K. Safety and advantage of no drain policy after thoracoscopic major lung resection. J Thorac Dis 2020;12(3):1136-1137. doi: 10.21037/jtd.2019.12.61

\section{Footnotes}

Conflicts of Interest: The author has no conflicts of interest to declare.

Ethical Statement: The author is accountable for all aspects of the work in ensuring that questions related to the accuracy or integrity of any part of the work are appropriately investigated and resolved.

Open Access Statement: This is an Open Access article distributed in accordance with the Creative Commons Attribution-NonCommercial-NoDerivs 4.0 International License (CC BY-NC-ND 4.0), which permits the noncommercial replication and distribution of the article with the strict proviso that no changes or edits are made and the original work is properly cited (including links to both the formal publication through the relevant DOI and the license). See: https://creativecommons.org/licenses/by-nc-nd/4.0/.

\section{References}

1. Miyazaki T, Nagayasu T. No drain policy for "ultimate" enhanced recovery after surgery. J Thorac Dis 2019;11:S1900-2.

2. Li P, Li S, Che G. Role of chest tube drainage in physical function after thoracoscopic lung resection. J Thorac Dis 2019;11:S1947-50.

3. Ueda K, Haruki T, Murakami J, et al. No Drain After Thoracoscopic Major Lung Resection for Cancer Helps Preserve the Physical Function. Ann Thorac Surg 2019;108:399-404.

4. Murakami J, Ueda K, Tanaka T, et al. The Validation of a No-Drain Policy After Thoracoscopic Major Lung Resection. Ann Thorac Surg 2017;104:1005-11.

5. Paul S, Jalbert J, Isaacs AJ, et al. Comparative effectiveness of robotic-assisted vs thoracoscopic lobectomy. Chest 2014;146:1505-12. 